\title{
Blockchain: Future of Accounting Education
}

\author{
Dr Munshi Samaduzzaman (“corresponding author") \\ Accounting Lecturer/Unit Coordinator, Central Queensland University, Sydney Campus \\ 400 Kent Street, Sydney, NSW 2000, Australia \\ Tel: 61-4-4928-9121_Ｅ-mail: m.samaduzzaman@cqu.edu.au
}

\author{
Received: August 1, $2020 \quad$ Accepted: Sep. 27, $2020 \quad$ Published: December 1, 2020 \\ doi:10.5296/ajfa.v12i2.17679 URL: https://doi.org/10.5296/ajfa.v12i2.17679
}

\begin{abstract}
Blockchain will be the future of accounting education. Triple entry accounting system is here, and shared ledger has been considered. From the shared ledger different parties can access transactions. As our discussion reveals that distributed ledger, Smart contract and Blockchain are three important elements in the triple entry accounting system. As a result, blockchain technology is helping the upgrading the process of education system. Blockchain technology is a peer to peer communication that allows participants to secure the settlement of transactions, achieve the transactions and transfer of assets at low cost. With certain advantages there are disadvantages too. Based on performance and acceptance, it is clear that in future the implication of blockchain technology would be developed. The concept of triple accounting has introduced the new way of accounting work replacing accounting standard formula. The blockchain technology eliminates the involvement of third party, maintain transparency and charges low transaction cost. It will save money and time of people as it is secure and due to decentralization, it is not controlled by one single entity. Due to the decentralization, every user of the network can see the file. So, blockchain should the part the Accounting Education in future.
\end{abstract}

Keywords: Blockchain, Cryptocurrency, Bitcoin, Accounting, Education 


\section{Introduction on Blockchain Technology \& Education}

According to Yang, Li, Wu \& Zhao (2017), blockchain technology is regarded as another innovative technology after cloud computing, internet of things and big data, which has received high concern by governments, financial institutions and technology enterprises. With the creation of Bitcoin (a cryptocurrency), blockchain technology concept was appeared. Blockchain technology is a technical scheme to maintain a huge and trusted database by means of confidence and high faith. Blockchain technology is not only important for financing but also has a great ability in education. It is assumed that the blockchain technology is important to upgrade the process of the educational system. With advancement of technology and trust built into technology, blockchain can help transform higher education and provide alternative version to secure and guarantee lifelong learning. With the help of artificial intelligence and improved technology, personalised learning is improving learning outcomes for students at all level. As it is easier to keep track and store information, blockchain technology could be used to upgrade the library. Blockchain technology includes learning behaviour in class, macro educational background, and micro academic project experience and many more. Blockchain technology contributes to minimize academic degree fraud as blockchain distributed ledger is unable to change and is reliable.

\section{About Blockchain}

This technology allows data to be stored and exchanged on a peer-to-peer basis. Blockchain data can share consulted and secured thanks to consensus-based algorithms. As it is being used in a decentralized manner, it removes the need for "trusted third parties" (Cachin, 2016). Blockchain is the chain of blocks where information of the transactions is being kept. However, blockchain is not fully secure, but it is being used for keeping various information to avoid fraudulent activities. The only con in the blockchain is when a block has been added to the last it is difficult to change or delete the blocks. It has been needed hackers to change the blocks. Blockchain emerged from the amalgamation of two concepts.

\subsection{Asymmetrical Cryptography}

It allows users who are unknown to each other to exchange encrypted information. The system is based on a public key that can be made available to all that allows encrypted data to be sent to a third party. Now a third party gets access to the encrypted data to a private key. Here the public key is similar to a bank account number and on the other hand, a private key that remains secret acts as the password for the same bank account number (Kosba et al., 2016).

\subsection{Distributed IT Architecture}

This system is a series of different independent computers. That connects to a network and can communicate with each other. The blockchain network is a peer to peer distributed system. Here, information is to be shared among the different users.

\section{Cryptocurrency}

According to Harwick (2016), a cryptocurrency is a method of representing virtual "coins" and providing secure ownership. A cryptocurrency is a digital currency that is created and managed 
through the use of advanced encryption techniques known as cryptography (Barone, 2019). A cryptocurrency is a digital or virtual asset which plays a huge role as medium of exchange. With the creation of Bitcoin, cryptocurrency has brought immense change from being an academic concept to virtual reality. Cryptocurrency is built with cryptographic protocols through advanced techniques which make transaction secure and difficult to fake.

There are various cryptocurrencies which are being popular in circulation today. Few of the popular cryptocurrencies are explained below:

\subsection{Bitcoin}

It is a digital currency launched in 2009. It is the most original and widely used cryptocurrency. Bitcoin is operated by decentralized authority and is not backed by any country's government and central bank. It is a virtual currency used for trading goods and services with vendors who accepts Bitcoins as payment. The value of Bitcoins can fluctuate quickly just as with stocks.

\subsection{Bitcoin Cash (BCH)}

The hard fork of the original Bitcoin, which was launched in 2017, has become one of the successful and most traded cryptocurrencies. Bitcoin cash has an $8 \mathrm{MB}$ blocksize compared with $1 \mathrm{MB}$ for the original Bitcoin which allows more transactions to be processed faster. It takes place as the result of misunderstandings and arguments between developers and miners.

\subsection{Litecoin (LTC)}

It was among the initial cryptocurrency followed by Bitcoin which was launched in 2011. Litecoin is a peer to peer cryptocurrency where creation and transfer of the coin is based on open source global payment network and is not managed by any central authority. It is also referred as "silver to bitcoin's gold" or "bitcoin's little brother" because Litecoin is like Bitcoin in many ways. It offers a faster transaction confirmation as it has a faster block generation rate.

\subsection{Ethereum (ETH)}

It is a decentralized software platform which was launched in 2015. It enables distributed applications to be built and run without any downtime, fraud and interference from a third party. Instead of mining for Bitcoin, miners can earn ether. Ether is a type of crypto token that fuels the Ethereum platform. The bitcoin blockchain offers one particular application that enables online bitcoin payments whereas the Ethereum blockchain focuses on operating the programming code of any decentralized application.

\subsection{Ripple $(X R P)$}

It is a global settlement network that offers instant and low-cost cross-border payments which was launched in 2012. It enables banks to settle international payments in real time with end to end transparency and at minimal cost. Ripple's consensus ledger is unique as it doesn't require mining and reduces the usage of computing power and minimizes network latency. It is one of the most convincing digital currencies among financial institutions for cross-border payments. 


\subsection{NEO}

It is the first open source cryptocurrency which was launched in China as AntShares in 2014. It is the largest cryptocurrency which aims to the development of smart contracts and assets on its platform. It targets to be the platform of choice for the new smart economy.

\section{Bitcoin}

According to Swan, (2015) Bitcoin is a digital currency or an online payment system in which encryption techniques are used to regulate the generation of units of currency and verify the transfer of funds, operating independently of a central bank. Bitcoin is the first crypto currency invented by an unknown person Satoshi Nakamoto in 2009. It is a type of electronic cash. So, it doesn't have any physical depiction. Bitcoin can also be defined as a virtual monetary unit. A unit of bitcoin is divisible and can be further divided into 100 million "satoshis" which is the smallest fraction of a bit coin. A bitcoin blockchain is often referred to as the ledger of the bitcoin system because it is a data file that carries the records of all past bitcoin transactions including the creation of a new bitcoin units. Bitcoin is very popular among the people.

Bitcoins are totally virtual coins designed to be self-content for their worth. There is no need for banks to move and store the money. Bitcoins are just like physical gold coins they possess the same value and trade just as if they are nuggets of gold in the owner's pocket. It can be used to purchase goods and services online or one might tuck them away and hope that their value increases over the years. Bitcoins are always transacted or traded from one personal wallet to another, where a wallet is a personal database that is stored on a computer drive, smartphone, tablet, or somewhere in the cloud. Bitcoins cannot be forged because it is very computationally intensive to create a bitcoin and hence, it is not financially worthy for the counterfeiters to manipulate the system. Bitcoins are generated in the market from the bitcoin mining. It has been assumed that bitcoin is very safe to use, but it has some trust issues between the parties who are dealing with bitcoin.

Bitcoins can use in various fields to raise funds for a start-up of new business. There is an example of exchange currency by Böhme, Christin, Edelman, \& Moore, (2015) that in spring 2012, the Japan-based Mt. Gox exchange served over 80 percent of all Bitcoin transactions. However, Mt. Gox collapsed in early 2014 and reported in its bankruptcy filing "losing" 754,000 of its customers' bitcoins worth approximately $\$ 450$ million at the time of closure (Abrams, Matthew, and Tabuchi 2014). In March 2015, the seven largest exchanges were BTC China, OKCoin, Huobi, Bitfinex, LakeBTC, Bitstamp, and BTC-e, which jointly served more than 95 percent of all bitcoin trade from October 2014 to March 2015.

\section{Implementation of Blockchain Technology in Accounting And Financial Services Industries}

As opined by Yeoh (2017), blockchain accounting technology has been used in case of identifying the transfer of ownership of assets and to maintain a ledger of financial information. This is because transparency of financial information is essential for ensuring accounting activities. This technology helps to reduce cost of maintaining ledger and provides certainty over the history of assets. Actually, blockchain has been used to allocate financial resources 
effectively, which reduces the ledger maintenance cost. As mentioned by Ducas \& Wilner (2017), the scope of accounting has been increased by blockchain. The reason is this technology helps to eliminate reconciliations and ensures the transaction history. As informed by Michal, Cohn \& Butcher (2018), blockchain technology has been used in resource planning and valuation rather than book recording. The effective planning of resource allocation increases efficiency level of accounting and financial service profession by developing cost strategy. Hence, in case of developing the performance of financial service industry, blockchain technology has a significant role. As trust and transparency in accounting has been ensured by blockchain, the acceptance of this technology is increasing day by day.

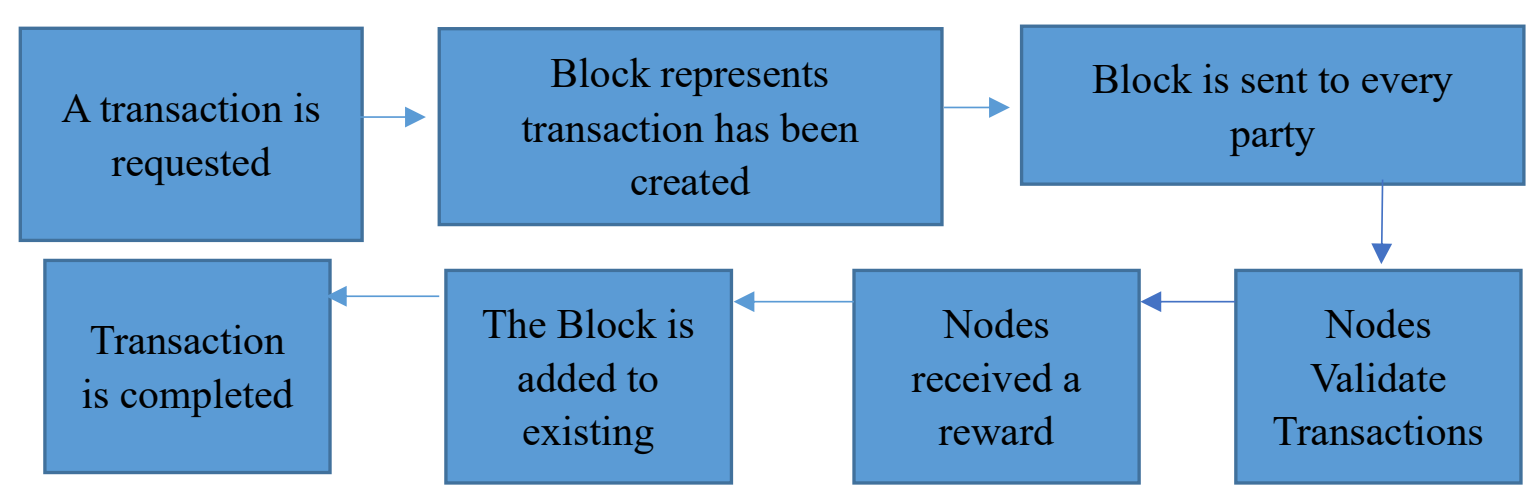

\section{Block Chain Mechanism}

According to Ducas \& Wilner (2017), blockchain technology helps to convert fiat to digital currency with the help of peer to peer protocol. Every peer has a list of transactions and validation of a transaction can be made through consensus. As stated by Michal, Cohn \& Butcher (2018), blockchain technology enhances business process in the financial service industry. It is done by sharing data in a transparent and effective manner. The implementation of this technology has completely changed the performance of financial service industry. This is because the digital cash transaction system has been completely developed based on blockchain throughout the world. This method has been used in case of the back-office operation for improving security and transparency of work. In order to develop capital market performance and enhancing external audit process, blockchain has pivot role. The technology helps to improve performance in financial industry by enhancing the mechanism. The development in the standard of performance in financial market has increased the number of transactions. This technology is really essential in the field of audit and accounting. As the performance inside the audit process has been significantly developed by blockchain, the acceptance of the technology is also increasing. Hence, the overall analysis reveals that blockchain has remarkable contribution in case of increasing value of digital assets and lowering the associated cost.

\section{Advantages of Blockchain Technology}

Chen, Xu, Lu \& Chen (2018) opined that blockchain technology allows participants to secure the settlement of transactions, achieve the transaction and transfer of an asset at a low cost. Some advantages of blockchain technology are described as follows: 


\subsection{Decentralization}

Blockchain technology allows individual transaction to have their own authorization to implement their constraints instead of running huge data center and verifying transactions through the same network. It ensures that if any information gets leaked, only a small amount of data will be compromised not the entire network.

\subsection{Reliability}

Due to the decentralized networks, the failures of a single network doesn't affect the operation of the whole network. This ensures the high reliability of the applications built on blockchain technology as it does not have a central point of failure.

\subsection{Transparency}

One of the primary reasons blockchain is exciting to businesses is that it is an open source network. Any changes in this network is publicly viewable by all parties involved creating transparency. All the transactions in this network are transparent to all the parties and are immutable i.e. they cannot be changed or deleted.

\subsection{Trust}

Blockchain technology acts as a new trust holder among the users with decentralized ledger. As it is a user controlled the network and there is transparency in the whole network, users can trust that the transactions will be executed exactly as the protocol commands.

\subsection{Enhanced security}

Blockchain is more secure than any other record keeping system. The information and data are stored across a network of computers instead of one single computer which makes difficult for hackers to hack the information or data. Blockchain technology can help prevent fraud and unauthorized activity by protecting sensitive data and information of business and company.

\subsection{Lower transaction costs}

For most of the businesses, reducing cost is priority. Blockchain technology allows peer to peer a transaction without the involvement of third party. By eliminating third party for transaction process, blockchain reduce transaction fees to the user or businesses (Song, Shi, Xu \& Gill, 2016).

\subsection{Faster transaction settlements}

With the traditional paper process, trading anything is time consuming. Transactions done through banks can potentially take days for final settlement. Banks and other financial institutes are only open during normal business hours, five days a week. Blockchain transactions can make the transaction faster as there are no intermediaries involved and is open 24 hours a day, seven days a week.

\section{Disadvantages of Blockchain Technology}

In spite of all the advantages discussed, the blockchain also has some disadvantages and 


\section{1) Macrothink}

Asian Journal of Finance \& Accounting

ISSN 1946-052X

2020, Vol. 12, No. 2

challenges. The disadvantages of the blockchain technology are explained below:

\subsection{Complexity}

As this is an advanced technology, non-technical people or old generation people cannot understand it easily. It has made cryptography more mainstream so without the proper understanding of the process, it is not possible to have a trustworthy service.

\subsection{Size of Blockchain}

With every new transaction happening in blockchain, data are being recorded so the size of the blockchain grows every second. Some famous cryptocurrencies like Bitcoin blockchain and Ethereum blockchain are of huge size so implementation of such blockchain is critical. Blockchain ledger can grow larger in due course. Size and storage remain a huge barrier for any application built on the blockchain.

\subsection{Costs}

The high cost is the big disadvantages of the blockchain. Blockchain offers remarkable savings in transaction costs and time but the high initial capital cost could be the restriction (Golosova \& Romanovs, 2018).

\subsection{Large energy consumption}

Blockchain is the high energy consumption network. The consumption of energy is required to maintain a real time ledger. Blockchain network requires nodes to run and nodes are giving time and energy to the network to run efficiently. The blockchain users are attempting thousands of transactions per second using extensive amount of computer power.

\subsection{Privacy}

Blockchain transactions might appear private since it is not directly attached to your identity, but as they are recorded in the public ledger personal data cannot be addressed in this platform without cyber security concerns (Song, Shi, Xu \& Gill, 2016).

\subsection{Security}

There is one security flaw in Bitcoin and other blockchain i.e. attack of 51\%. If two or more miners are working as nodes or calculating the hash of the blocks at the same time, they will get same results. The network will tell a lie and the lie will become the truth.

\subsection{Human error}

As the data in the blockchain cannot be altered or deleted, the database needs to be $100 \%$ sure and correct. Most of the blockchain are accessed through Private Key. If the private key is lost, it is impossible to access the network. So, the data must be accurate.

\section{Futures of Blockchain Technology}

As opined by Kokina, Mancha \& Pachamanova (2017), on the basis of performance and acceptance, it is clear that in the future the implication of blockchain technology would be 
developed. A blockchain based asset identity platform would be helpful to collect and share data for both virtual and physical assets. It is essential for gathering recording information in respect to virtual and physical assets separately. As per Yeoh (2017), it has been projected that more than 20 billion IOT devices would be existed by 2020. This technology would provide reliable and secure mechanism to IOT devices for making transactions effectively. Blockchain would help to provide unique identity to virtual assets. This is because; technology has the power to maintain virtual and physical assets separately. As mentioned by Ducas \& Wilner (2017), international trade would be conducted through blockchain by 2030 . The development of international trade would help to increase standard of living of people all over the world. Hence, the technology-based performance would help to develop the international living standard. The quality of world trade would be improved, and the entire business world would depend on technology. As opined by Nowiński, W., \& Kozma (2017), the blockchain technology would provide effective business value to the international supply chain. This is because the world trade has been conducted currently through chaotic business relationships among parties.

As stated by Ducas \& Wilner (2017), the government of various nations would adopt virtual currency within 2030 based on the implementation of blockchain technology. This is because virtual currency system helps to reduce the settlement time and ensures accuracy. Hence, for enhancing financial transactions internationally, blockchain has vital role. Continuing in the same vein, Nowiński, W., \& Kozma (2017) have opined that the efficiency of the performance of the government of different nations would be developed. The reason is quick settlement can be made in case of financial transactions. According to Michal, Cohn \& Butcher (2018), government policies can be easily adopted throughout the world based on the blockchain. As a result, the international relationship among various nations would be improved. Hence, the implementation of this technology would change the international scenario within few years. The technology-based performance would make the entire business world faster. As a result, the business performance would be more dynamic, and competition would be increased.

\section{Triple Entry Accounting}

The concept of triple entry accounting has been developed by Yuji Ijiri, a professor of Carnegie Mellon University. This concept has introduced the new way of accounting work replacing standard accounting formula (Simoyama, Grigg, Bueno \& Oliveira, 2017). As stated by Dai \& Vasarhelyi (2017), the triple entry accounting is important in case of strategic decision-making process. This is because; this accounting technique considers the outside parties in accounting entries. Actually, third entry has been made in case of triple entry accounting for maintaining transparency in accounting activities. The concept of this accounting system is new in the market which considers cryptographic technology. The reason is cryptographic technology helps to maintain accounting information easily. In case of this accounting system the linkage has been made between two double entry accounting systems. The linkage is made to make it publicly available to see transactions. In case of implementing triple entry accounting system, blockchain has been considered. As mentioned by Simoyama, Grigg, Bueno \& Oliveira (2017), the triple entry accounting is essential for developing the business performance effectively. This is because transparency can be clearly maintained in accounting activities and right 
policies can be adopted. This accounting technique considers double entry plus cryptography and distributed ledger (blockchain) which ensures the performance of accounting activity.

As opined by Wang \& Kogan (2018), the triple entry accounting system helps to improve reliability in accounting system. This is because; flawless recording of transactions can be made. Furthermore, the transparent overview of the transactions can be identified easily based on triple entry accounting policy. Continuing in the same vein, Dai \& Vasarhelyi (2017) have informed that implementation of the concept of blockchain has developed the acceptance of triple entry accounting. For accessing, updating and sharing accounts in the context of business relationship, this accounting technique has effective role. This is because; the concept of distributed ledger has been used in triple entry accounting system. As mentioned by Wang \& Kogan (2018), the important concept which has been used in triple entry system is smart contracts. The technique helps to develop the performance of financial service industry. The reason is that the use of technology enhances the productivity in the financial service sector. The main advantage of the triple entry accounting system is here shared ledger has been considered. From the shared ledger different parties can access transactions. Hence, the discussion reveals that Distributed ledger, Smart contract and Blockchain are three important elements in the triple entry accounting system.

\section{Accounting Standards at Both International And Domestic Level}

Blockchain is an accounting technology. It is concerned with transfer of ownership of assets and maintaining a ledger of accurate financial information. With the rapid growth of blockchain technology, the problem of lack of standards is also increasing. Blockchain technology promotes development of international standards in the field of distributed bookkeeping technology (Vidrih, 2018). As blockchain can hold currency transactions without any third party, it can hold transactions of any kind of property: shares, bonds, mortgage and others. Lazanis (2015) emphasizes that blockchain eliminates the need of banks, insurance companies or any financial institutions if company voluntarily publishes its data and transactions on blockchain network. As for domestic level, blockchain technology has been increasing economic and accounting standards. Government should start thinking how blockchain technology can be implemented in regard to monetary policy. According to the needs and conditions of the country, a cryptocurrency can be created that will make the transactions easy and make the accounting process convenient. If new cryptocurrency is created to act as a country's official currency, it would be a great development for the country and its monetary policy.

According to Wetzel (2018), the economic struggles of developed and developing countries require a blockchain technology as it can sufficiently provide a new economic structure that would assist developing and developed countries with economic setbacks. It showcases the revolutionary potential that blockchain have in improving the standards of individuals and governments. The blockchain as a source of trust can be helpful for the accounting structure. Modern accounting system is based on a double entry system. But with the implementation of blockchain technology, it can replace the method of double entry and make the transactions easier and faster. Blockchain technology can help improve the accounting process and makes 
the transaction secure and easier.

\section{Blockchain Technology should be the part of Accounting \& Finance Related Course}

Blockchain is mainly use for maintaining the ledger of all transactional data of assets and the wallet balance. Blockchain can be used in accounting finance related course, as it is transparent and there is low transaction cost in transferring bitcoin in the ledger and eliminate the thirdparty involvement. If a firm were to all voluntarily post of its business transactions on a blockchain, with a permanent time stamp on each transaction, the firm's entire ledger would be instantaneously visible and anyone could aggregate the firm's transactions into income statements and balance sheets in real-time (Byström, 2016). The blockchain technology can be used in any market, as it has no master file like only one person will not get the access of the data, but every user can work with the data. So, if any one wants to change the data they must ask for the majority's opinion. Through blockchain technology, companies can move forward a step-in accounting by keeping separate records of transaction receipts, by writing directly in the joint register to enduring the accounting records by interlocking system. Therefore, the data and information are cryptographically sealed and safe from the fraudulency or distortion.

Blockchain is very useful for financial sector as the blockchain technology is based on transparency, decentralization and immutability (Tapscott, \& Tapscott, 2017). It helps financial institutions to create direct link with each other without involving a third party. Digitization helps the data to be kept more securely and maintained high privacy. The aspects of blockchain technology for financial sector are discussed below.

\subsection{Transparency}

Blockchain technology helps people by giving privacy while doing transactions but it is transparent. While people transact it keeps person's original identity hidden by complex cryptography and dhow the public address to others. Therefore, it helps people to be honest while using blockchain technology for transaction.

\subsection{Decentralization}

Decentralization is used in blockchain technology to make the transactions easier. Here involvement of third party is not needed. One can easily send money to the other person without going to bank. Bitcoin is the great example of blockchain technology.

\subsection{Immutability}

The advantage of blockchain is, once data or information is entered in the blockchain it cannot be changed or tampered. So, it is very useful for companies to keep the financial records in the blockchain.

\section{Blockchain Technology as a Future part of Accounting \& Business-Related Education}

As opined by Mougayar (2016), blockchain technology has impact on the transaction recording, authorization and reporting process. This is because the accuracy in the transactions can be easily maintained on the basis of this technology. Hence, in future it is essential to include the blockchain technology in business or accounting related education. The concept of this method 
would help to identify people the importance of blockchain in accounting and business performance. As mentioned by Dai \& Vasarhelyi (2017), in case of enhancing financial activities and taxation process blockchain technology has significant role.

This is because the accuracy in the performance of financial industry has been developed by this technology. The banking sector has become faster due to the use of blockchain technology. This has developed transparency and quality of service. Currently, businesses spend hefty amount of money in auditing. With the ease of access in blockchain technology, this is likely to decrease. As the blockchain gain more authority in the world of finance, the nature of accounting for cryptocurrency is subject to change.

Many companies and industries adopt blockchain based revenue streams. Similarly, accounting based blockchain will need to adapt. As companies adopt this new technology, less auditing manpower and more experienced professionals with vast knowledge is required. The implication of this shift in accounting strategy may be confusing for modern businesses. The replacement for double entry accounting method could be possible as it allows secured and verified information to be stored and accessed by multiple parties from multiple locations. Accountants and auditors working may not know about the techniques and procedure to operate them. So instead of firing them, the business owner can educate them on cryptocurrency and blockchain technology. The changes and developments in this technology should be tracked and discussed with the accountant, financial advisor and auditor of the business. They can help business to understand further implications and show the actions to be taken for increasing profit and reducing cost (Welker, 2018). With proper knowledge of the blockchain technology and its implications, a business can take advantage of saving time and money in the auditing process. To receive and verify data and information easily, an instantly accessible decentralized ledger could be of great help. This technology opens up new opportunities. Accountants' and auditors' skills will need to expand to include an understanding principle features, functions and implications of blockchain technology. But sadly, some auditors and accountants who fail to understand and adopt this technology may lose his/her career.

\section{Cost \& Benefit Analysis}

Blockchain is mainly used as an alternative for the traditional payment system. Blockchain systems can be successfully executed in the market like hospitals, financial institutions, companies, economy, software engineering etc. (Yli-Huumo et al., 2016). The blockchain technology is maintained by a highly secured peer-to-peer network. It has a high level of resistance to accommodating data available in the blockchain. The computers that are involved in this network have the consent that the transactions are valid only before the entry of a new ledger. Due to the decentralization, every user of the network can see the file. In addition, no one has the power of computation to the networks. Therefore, individuals form a group to increase computational power. They thought of an alternative program of blockchain. All the functions of blockchain are based on a hash function. The hash rate is used for the calculation of measurement of the power of the network. Blockchain is very easy to operate. The changes can be done only when a new block added to the chain. It is difficult to change the data in the previous block, as it has been already said that the data in the blocks cannot be changed or 
deleted. Therefore, the company needs to ask a hacker to delete or change the data it is costly.

Recently, the cryptocurrency is very popular among the people to raise capital for business. However, that does not mean that any business could put a thin layer of blockchain and flourish their business by raising capital. Many businesses are now digitizing their operation and services. Blockchain can be used in many businesses to reduce its cost. Previously the cost of the transaction of bitcoin was free. However, after 2016 it charges a minimum of $\$ 0.20$ each transaction. It can only store 80 bytes of data in a block and can process 7 transactions per second (Cai \& Zhu, 2016).

\section{Limitations of the Blockchain Research}

The study is not free from the limitations. This research is totally based on secondary data. The data, that is available may lack of authenticity. The data available over the internet was depended on other companies. The data and information may be general, or for some particular company. Lack of prior studies on this particular topic is also a major concern for this study. At the same time, thorough analysis has done to find and verify the results.

\section{Conclusion}

In the nutshell it can be concluded that, blockchain technology is not only important for financing but also has great ability in education. It helps in upgrading the process of education system. Blockchain technology is a peer to peer communication that allows participants to secure the settlement of transactions, achieve the transactions and transfer of assets at low cost. With certain advantages there are disadvantages too. On the basis of performance and acceptance, it is clear that in future the implication of blockchain technology would be developed. The concept of triple accounting has introduced the new way of accounting work replacing accounting standard formula. This concept is important for strategic decision-making process. Blockchain technology is very useful to use in accounting and financial sector, but there are many obstacles need to overcome. Blockchain technology based on accounting sector is being adapted by many companies and businesses which could replace double entry accounting method. The blockchain technology eliminates the involvement of third party, maintain transparency and charges low transaction cost. It will save money and time of people as it is secure and due to decentralization, it is not controlled by one single entity. Due to the decentralization, every user of the network can see the file.

\section{References}

Barone, A. (2019). The future of cryptocurrency in 2019 and beyond.

Böhme, R., Christin, N., Edelman, B., \& Moore, T. (2015). Bitcoin: Economics, technology, and governance. Journal of Economic Perspectives, 29(2), 213-38. https://doi.org/10.1257/jep.29.2.213

Byström, H. (2016). Blockchains, real-time accounting and the future of credit risk modelling. Lund University, Department of Economics.

Cachin, C. (2016, July). Architecture of the hyperledger blockchain fabric. In Workshop on 
distributed cryptocurrencies and consensus ledgers (Vol. 310).

Cai, Y., \& Zhu, D. (2016). Fraud detections for online businesses: a perspective from blockchain technology. Financial Innovation, 2(1), 20. https://doi.org/10.1186/s40854-0160039-4

Chen, G., Xu, B., Lu, M., \& Chen, N. S. (2018). Exploring blockchain technology and its potential applications for education. Smart Learning Environments, 5(1), 1. https://doi.org/10.1186/s40561-017-0050-x

Dai, J., \& Vasarhelyi, M. A. (2017). Toward blockchain-based accounting and assurance. Journal of Information Systems, 31(3), 5-21. https://doi.org/10.2308/isys-51804

Ducas, E., \& Wilner, A. (2017). The security and financial implications of blockchain technologies: Regulating emerging technologies in Canada. International Journal, 72(4), 538562. https://doi.org/10.1177/0020702017741909

Golosova, J., \& Romanovs, A. (2018). The Advantages and Disadvantages of the Blockchain Technology. https://doi.org/10.1109/AIEEE.2018.8592253

Harwick, C. (2016). Cryptocurrency and the Problem of Intermediation. The Independent Review, 20(4), 569-588.

Kokina, J., Mancha, R., \& Pachamanova, D. (2017). Blockchain: Emergent industry adoption and implications for accounting. Journal of Emerging Technologies in Accounting, 14(2), 91 100. https://doi.org/10.2308/jeta-51911

Kosba, A., Miller, A., Shi, E., Wen, Z., \& Papamanthou, C. (2016). Hawk: The blockchain model of cryptography and privacy-preserving smart contracts. In 2016 IEEE symposium on security and privacy (SP) (pp. 839-858). IEEE. https://doi.org/10.1109/SP.2016.55

Lazanis, R. (2015). How Technology Behind Bitcoin Could Transform Accounting as We Know It.

Michal, J., Cohn, A. L. A. N., \& Butcher, J. R. (2018). Blockchain technology. The Journal, 1, 7.

Mougayar, W. (2016). The business blockchain: promise, practice, and application of the next Internet technology. John Wiley \& Sons.

Nowiński, W., \& Kozma, M. (2017). How can blockchain technology disrupt the existing business models?. Entrepreneurial Business and Economics Review, 5(3), 173-188. https://doi.org/10.15678/EBER.2017.050309

Simoyama, F. D. O., Grigg, I., Bueno, R. L. P., \& Oliveira, L. C. D. (2017). Triple entry ledgers with blockchain for auditing. International Journal of Auditing Technology, 3(3), 163-183. https://doi.org/10.1504/IJAUDIT.2017.086741

Song, W., Shi, S., Xu, V., \& Gill, G. (2016). Advantages \& Disadvantages of Blockchain Technology. 
Swan, M. (2015). Blockchain: Blueprint for a new economy. O'Reilly Media, Inc.

Tapscott, D., \& Tapscott, A. (2017). How blockchain will change organizations. MIT Sloan Management Review, 58(2), 10.

Vidrih, M. (2018). The Blockchain International Standardization Organization: China Will Dominate.

Wang, Y., \& Kogan, A. (2018). Designing confidentiality-preserving Blockchain-based transaction processing systems. International Journal of Accounting Information Systems, 30, 1-18. https://doi.org/10.1016/j.accinf.2018.06.001

Welker, B. (2018). What Blockchain Could Mean for the Future of Accounting and Business.

Wetzel, T. (2018). International \& Domestic Monetary Policy Reform with Blockchain and Cryptocurrencies.

Yang, X., Li, X., Wu, H., \& Zhao, K. (2017). The application model and challenges of blockchain technology in education. Modern distance education research, (2), 34-45.

Yeoh, P. (2017). Regulatory issues in blockchain technology. Journal of Financial Regulation and Compliance, 25(2), 196-208. https://doi.org/10.1108/JFRC-08-2016-0068

Yli-Huumo, J., Ko, D., Choi, S., Park, S., \& Smolander, K. (2016). Where is current research on blockchain technology? - a systematic review. PloS one, 11(10), e0163477. https://doi.org/10.1371/journal.pone.0163477

\section{Copyrights}

Copyright for this article is retained by the author(s), with first publication rights granted to the journal.

This is an open-access article distributed under the terms and conditions of the Creative Commons Attribution license (http://creativecommons.org/licenses/by/4.0/) 